\title{
Involving Males from Community in Family Planning Program
}

\section{Mashal Feroz Hirani and Sarmad Muhammad Soomar*}

Aga Khan University, Karachi, Pakistan

${ }^{*}$ Corresponding author: Sarmad Muhammad Soomar, Aga Khan University, Karachi, Pakistan, E-mail: soomarsarmad@gmail.com

Received date: August 13, 2018; Accepted date: September 27, 2018; Published date: October 03, 2018

Copyright: (c) 2018 Hirani MF, et al. This is an open-access article distributed under the terms of the Creative Commons Attribution License, which permits unrestricted use, distribution, and reproduction in any medium, provided the original author and source are credited.

Citation: Hirani MF, Soomar SM (2018) Involving Males from Community in Family Planning Program. Crit Care Obst Gyne Vol.4 No.3:12

\section{Editorial}

Proper spacing and limiting pregnancy is essential for maternal and child health. Life partner help is significant predictor to women especially on utilization of contraception. On the other hand, in our society male dominance hinders the use of family planning methods. As literature also supports involving men in family planning program can gradually improve outcome and gender equality [1].

The reason to talk about male dominance in regard to family planning is, because in clinical practice we encounter such cases in a huge quantity. While discussion with women in community we found that mostly Pakistani families are of huge size, mainly comprised of 4 to 5 children within an age difference of mostly 10 to 12 months. While inquiring about family we usually hear less cooperation of male partner. Women report that males don't want to wear condom as they feel very uncomfortable during coitus. Furthermore, women in community reveal that they wish to use known contraceptive methods for spacing but they can't anything alone. Also, many husbands took contraception in lieu of restricted by religion or culture. Moreover, women report unintended pregnancy and miscarriages as a result of lack of mutual support from partner and lack of use of contraception.

Many of them were educated and were aware that it is not good for health, understanding family planning and contraception can bring better health of them and better future for children. Few of these women from community were weeping while sharing their ill health and critical conditions after fourth or fifth pregnancy. Some of them were hospitalized for proper treatments and very seriously ill. Pakistan is one of the poorest and populated country in world due to barrier and perceptions of family planning including lack of knowledge and accessibility of contraceptives [2]. Male dominance is one of the barriers to family planning in our society. Decision making power is in the hand of men and traditionally they decide about contraceptive preferences and reproductive actions. Men usually have misconception and lack of knowledge about fertility preventive methods, as a result they refuse to utilize contraception and consider it inappropriate. One of the researches of pilot distribution also explains that most couples who were given condoms at no cost did not utilize them. The rationale for not using was they thought that condom use could cause impotence (Population reports, 1982). Additionally, this issue also increases because of communication gap between partners. As one study states $45 \%$ of married women didn't know what their husband's perspective is related to family planning or assumed that they disapproved it without asking to them [1]. Finally, the awareness and counseling programs only focuses women while neglecting men. The belief that family planning process is only women's need and keeping men aside, men feel uncomfortable and hesitate to seek help from such clinics and health care center.

As community nurses and midwives, working related to family planning our work should relate in developing strategies for counseling of both men and women and encourage them for a mutual and safe decision. In case couple is not ready to visit us and take suggestion, we have pattern to plan a home visit and talk to the couple especially about these important concepts. Researches done in South Asia supports that inclusion of men in family planning programs have improved approach and use of family planning in their region [3].

Also, home survey should be done in detail to identify these issues. If couples and families are not easy to share, provide those leads and make them comfortable to talk about these topics. Data should be collected and recorded appropriately and working should be accordingly. Afterwards, in community we should arrange group education involving all genders and sexes, in which we can tell about benefits and proper techniques of using family planning. Secondly, religious myth related to family planning can be resolved by engaging religious leaders in counseling sessions. For nurses and midwives themselves its necessary to understand why family planning is connected or argument in lieu of religion and how to address this kind of community concerns. Future more, media campaigns can be run to give short beneficial messages related to family planning. Lastly, we should understand the male nurses or midwives should also be included in reproductive medicine and they should be provided spots in community, especially in context of Pakistan to address men separately regarding sexual and reproductive health and family planning [4].

As health care providers we can play a vital role in empowering both sexes through knowledge, information and better understanding to improve maternal and child health indictors in Pakistan [5]. 


\section{References}

1. USAID (2013) Increasing Male Involvement in Family Planning in Jharkhand, India. Progress in Family Planning 2-3.

2. Azmat KS, Mustafa G, Hameed W, Ali M, Ahmed A, et al. (2012) Barriers and perceptions regarding different contraceptives and family planning practices amongst men and women of reproductive age in rural Pakistan: a qualitative study. Pak J Public Health 2.
3. USAID (2012) Increasing men's engagement to improve family planning programs in South Asia. Progress in family planning 2-5.

4. Greene M (2000) Involving men in reproductive health: contributions to development. Sexual and Reproductive Health and Millennium Development Goal 10-40.

5. WHO (2001) Programming for male involvement in reproductive health. Regional Advisers for reproductive health 3-10. 